\title{
Organização de Grupos de Trabalho em Projetos Utilizando Sistemas Multi-Agentes
}

\author{
Ryan Ribeiro de Azevedo ${ }^{1}$, Emanuel B. Santos ${ }^{1}$, Robson Godoi ${ }^{1}$, Fred Freitas ${ }^{1}$, \\ Marcelo José Siqueira Coutinho Almeida ${ }^{1,2}$
}

\author{
${ }^{1}$ Centro de Informática - Universidade Federal de Pernambuco (CIN-UFPE) \\ Caixa Postal 7851 -50732-970- Recife - PE - Brasil \\ \{rra2, ebs, rgam, fred\}@cin.ufpe.br \\ ${ }^{2}$ Coordenação de Informática - Centro Federal de Educação Tecnológica da Paraíba \\ Av. $1^{\circ}$ de Maio, 720, Jaguaribe, CEP 58015-430, João Pessoa - PB - Brasil \\ marcelo@cefetpb.edu.br
}

\begin{abstract}
This article proposes a Multi-Agents system to solve the problem of composing workgroups for projects using the framework JADE. Its functioning is based on the interaction protocol contract-net. The agents should negotiate with each other the vacancy on the projects and the winners of this negotiation will be the final result. The argumentation used in this negotiation process among the agents is based on the criterion of brevity to the conclusion of each activity of a certain project.
\end{abstract}

Resumo. Este artigo propõe um sistema Multi-Agentes para resolver o problema de composição de grupos de trabalho para projetos utilizando o framework JADE. Seu funcionamento é inspirado no protocolo de interação contract-net. Os agentes deverão negociar entre si as vagas nos projetos e os agentes vencedores desta negociação farão parte do resultado final. A argumentação utilizada neste processo de negociação entre os agentes é baseada no critério de brevidade para a conclusão de cada atividade de um determinado projeto.

\section{Introdução}

A composição de grupos de trabalho em projetos é algo complexo por avaliar diversos atributos tanto dos indivíduos como das atividades desenvolvidas por eles. É de extrema importância para as organizações compor equipes que executem seus projetos em menor tempo possível e com qualidade. Para auxiliar nessa tomada de decisão está sendo proposto um sistema que sugere a alocação de indivíduos em grupos de trabalho de acordo com as atividades, perfis e duração de um determinado projeto a ser desenvolvido.

O problema de composição de grupos é conhecido como manpower scheduling ou employee scheduling e é uma instância do problema clássico de scheduling (escalonamento) [Conway, Maxwell, e Miller 1967]. O employee scheduling consiste em organizar uma agenda com os horários dos funcionários. Para resolver esse problema são utilizadas técnicas de resolução de problemas por CSP (Constraints Satisfaction 
Problems), por meta-heurísticas como Algoritmos Genéticos e busca tabu, ou por técnicas de programação linear como o método Simplex [Shamblin e Stevens 1979]. Esses métodos procuram encontrar uma solução viável em termos de tempo para a organização. Esse tipo de solução se aplica as situações onde há restrições de tempo (horário dos funcionários) ou de recursos (ociosidade de máquinas).

Procurando uma forma alternativa de solucionar o problema de composição de grupos em projetos de forma não centralizada como os métodos citados anteriormente propôs-se usar sistemas Multi-Agentes no desenvolvimento dessa aplicação, considerada uma solução distribuída para o problema. O sistema objetiva obter uma solução viável cujo escalonamento resultante envolva o menor tempo possível ou um determinado tempo e uso da computação simbólica na tentativa de criar uma solução viável. O resultado do desenvolvimento é um sistema que recebe definições de projetos de uma dada organização e retorna a configuração de um grupo de trabalho que executaria tal projeto em menos tempo ou no tempo proposto.

Este trabalho está organizado da seguinte forma: na Seção 2 são descritos os trabalhos relacionados. A Seção 3 justifica a escolha e a relevância do tema e explica os objetivos do projeto, a Seção 4 foca em como a solução proposta esta estruturada, na Seção 5 é descrito o funcionamento do sistema, sua arquitetura e um exemplo ilustrativo, na Seção 6, pode-se visualizar os resultados alcançados a partir dos experimentos realizados. As conclusões e trabalhos futuros se encontram na Seção 7.

\section{Trabalhos Relacionados}

O problema de planejamento de escala de serviço para trabalhadores possui uma literatura bastante extensa e diversificada, sendo inclusive um tópico de estudo na área de Pesquisa Operacional. Embora não haja uma terminologia padrão entre todos os autores, esse problema é conhecido como manpower scheduling ou employee scheduling. Diferentes autores utilizam a mesma terminologia, porém suas pesquisas são direcionadas para subproblemas, como escalas para: enfermeiras, militares, condutores, entre outros.

Diversas técnicas são utilizadas para resolução de problemas com escalonamento de empregados, onde se procura encontrar uma solução viável em termos de custos para a organização, levando em consideração restrições de tempo ou de recursos, a seguir são apresentados algumas dessas técnicas.

No trabalho de [Chan e Weil 2002] é analisado o uso simultâneo de diferentes modelos de resolução, utilizando CSP (Constraints Satisfaction Problems), objetivando resolver problemas de escalonamento de empregados em larga escala. Na área de MetasHeurísticas pode-se citar: [Gröbner e Wilke 2001], onde os problemas da vida real relativos ao escalonamento de empregados são atacados através da aplicação de Algoritmos Genéticos Híbridos, onde são utilizados conhecimentos específicos de cada problema; e em [Glover e McMillan 1986], o problema de escalonamento de empregados é atacado com a integração da Ciência do Gerenciamento e da Inteligência Artificial, através de busca tabu.

De maneira mais abrangente, [Tien e Kamiyama 1982] faz uma revisão e comparação dos diversos algoritmos disponíveis de manpower scheduling, baseado no 
framework dos cinco estágios, onde problemas gerais de escalonamento são decompostos em cinco subproblemas (estágios) separados, porém relacionados.

As vantagens do trabalho proposto em relação aos trabalhos relacionados citados acima são: contribuição do trabalho apresentado como um Sistema de Apoio à Decisão no planejamento de projetos em empresas e composição de equipes de projeto, algo fundamental na implementação de Sistemas de Informação em relação à alocação de funcionários, conforme perfil determinado para participação no desenvolvimento dos projetos. O uso da tecnologia e ambiente distribuído de Sistemas Multi-Agentes, adequada e eficiente neste domínio para alocação de recursos.

\section{Contextualização}

Para restringir o escopo da aplicação resolveu-se utilizar como estudo de caso a organização de grupos de trabalho em empresas que trabalham orientadas a projetos de software. Nesse modelo de organização os funcionários são alocados dinamicamente em grupos para trabalhar nos projetos assumidos. Cada projeto possuirá características individuais relevantes para a composição dos grupos de trabalho, tais como: tipo de projeto; duração do projeto; atividades; papel do profissional necessário para cumprir uma determinada tarefa.

A composição dos grupos de trabalho se dará através da representação das características dos indivíduos que são relevantes ao desenvolvimento do projeto. Características como a experiência, especialidade e disponibilidade de tempo, por exemplo, são relevantes na escolha de qual indivíduo fará parte do grupo. O sistema representa os funcionários da empresa como agentes. Quando um novo projeto chegar à organização, a decisão de quem trabalharia em determinado projeto seria tomada pelos agentes. Essa decisão seria emergente dos agentes e viria da negociação entre eles.

\section{Arquitetura de SMA proposta}

Para desenvolver a aplicação, está sendo utilizado um sistema Multi-Agentes com agentes cognitivos. Como os agentes não têm que executar tarefas complexas, que exijam grande capacidade cognitiva, a arquitetura dos agentes é simplificada para se adequar melhor ao problema. Essa economia cognitiva não significa, no entanto, que os agentes serão incapazes de ter comportamentos ditos inteligentes. Os estados internos dos agentes são usados para definir a situação dos agentes em processos de tomada de solução.

Como o projeto utiliza mecanismos de interação e negociação para obter uma solução é necessário evitar falhas como impasses e deadlocks para que o sistema possa garantir uma solução. Não é desejável que a negociação entre agentes se estenda infinitamente, por isso será utilizada uma argumentação simples e de duração limitada em uma negociação.

O sistema foi implementado na linguagem de programação Java [Gosling e McGilton 1996] por prover um conjunto considerável de bibliotecas que podem vir a ser utilizadas e desenvolvido na plataforma de desenvolvimento de sistemas Multi-Agentes JADE (Java Agent DEvelopment Framework) [Bellifemine et al 2003]. O uso de JADE permite abstrair alguns elementos do desenvolvimento como o controle de concorrência e o mecanismo de troca de mensagens. 


\section{Descrição do Sistema}

O modelo de organização utilizado pelo sistema é composto por projetos e funcionários. As características de cada um desses elementos são armazenadas em arquivos XML Extensible Markup Language. Quando a aplicação é iniciada, os elementos são lidos e representados através de instâncias de projeto ou funcionário, sendo o último representado por agentes.

Para fazer a escolha de quais agentes farão parte de cada projeto, será utilizado o protocolo de interação contract-net [FIPA 2002], onde a vaga em um projeto será conseguida através da avaliação das propostas dos agentes interessados. Dessa forma a decisão seria emergente do conjunto de agentes. O funcionamento básico do sistema é apresentado na Figura 1.

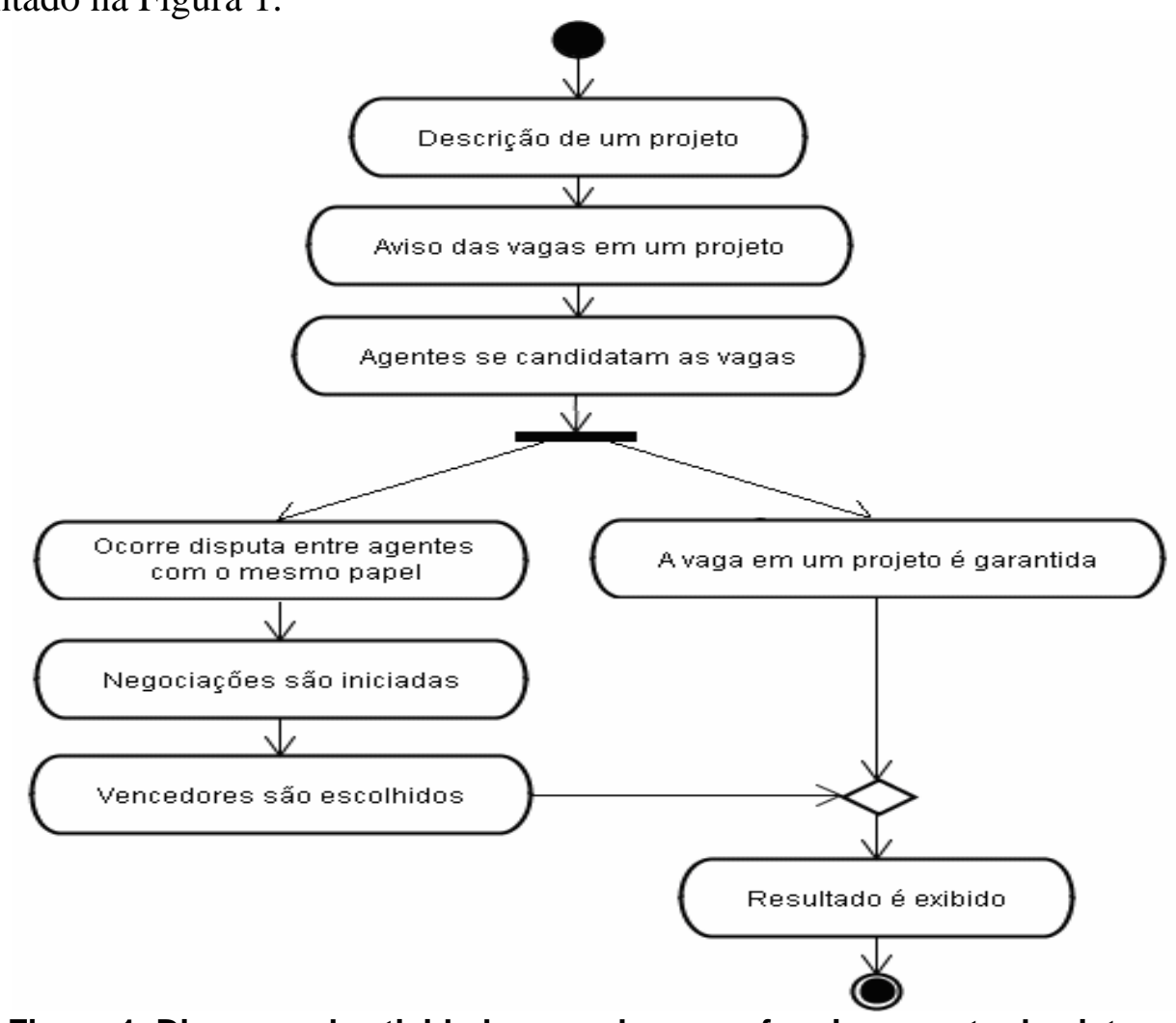

Figura 1. Diagrama de atividades que descreve funcionamento do sistema.

A seqüência de execução inicia com a entrada dos dados do projeto, em seguida os agentes recebem a notificação de existência de um novo projeto. Os agentes procuram submeter seus perfis a um leiloeiro que identificará conflitos entre agentes com os mesmos papeis. Depois de identificados os conflitos, os agentes passarão a negociar quem ficará com a vaga no projeto. $\mathrm{O}$ vencedor da negociação passa a fazer parte da solução final. Caso não haja disputa, a vaga é diretamente atribuída ao agente que será responsável por executar determinada atividade no projeto.

Para garantir que a solução não será apenas uma solução aleatória, as negociações entre os agentes serão baseadas em critérios que poderiam influenciar o desenvolvimento de um projeto. Para representar isso serão utilizadas restrições de produtividade em função do tempo. O tempo para desenvolver uma tarefa pode ser diferente para agentes com características diferentes. Um agente com mais experiência 
ou um histórico favorável, por exemplo, levaria menos tempo para executar uma determinada atividade. Os atributos dos agentes são a base para a negociação, cada atributo é usado como argumento e deve ser interpretado pelos agentes que estão participando de uma negociação. Caso um agente identifique que suas características não são suficientes para ganhar uma negociação ele desiste da negociação e dá a vaga ao outro agente.

Para representar os projetos foram considerados os seguintes atributos:

- TIPO DE PROJETO: cada projeto será classificado por tipo, representando o seu domínio (Indústria, Comércio, Governo);

- DATA DE INÍCIO: representando quando o projeto será iniciado. Esta informação será expressa em termos de unidade de tempo, a partir da entrada do projeto no sistema;

- DURAÇÃO: representando a quantidade máxima de unidades de tempo, esperada para a conclusão do projeto, a partir da DATA DE INÍCIO;

- ATIVIDADES: representando as tarefas a serem executadas por cada funcionário da organização. Cada atividade será expressa em termos de:

- DESCRIÇÃO DA ATIVIDADE

- INICIO DA ATIVIDADE: indicando a quantidade de unidades de tempo passadas da DATA DE INÍCIO.

- PRAZO DE CONCLUSÃO: indicando a quantidade de unidades de tempo necessárias para a conclusão desta ATIVIDADE.

- PERFIL: indicando o papel do profissional necessário para desempenhar a ATIVIDADE.

- DESCRIÇÃO: descrição do projeto a ser desenvolvido.

É apresentado na Figura 2 a representação adotada para os projetos, incluindo seus atributos.

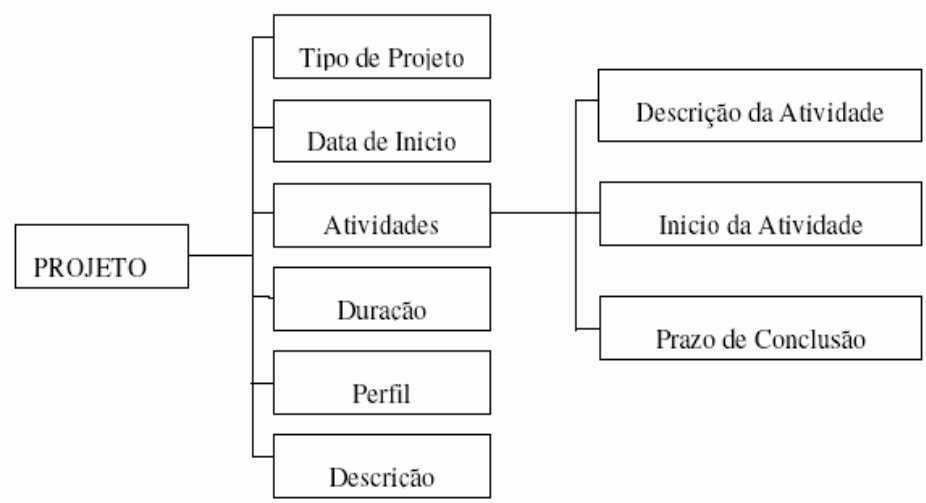

Figura 2. Representação dos atributos referentes aos projetos.

Cada funcionário será representado no sistema através de um agente com os seguintes atributos internos:

- NOME: indica o nome do suposto funcionário. 


\section{Simpósio Brasileiro de Sistemas de Informação}

- PAPEL: indicando a função a ser exercida pelo profissional (Analista de Negócios, Engenheiro de Software, Gerente de Projeto, Desenvolvedor).

- EXPERTISE: representando o grau de experiência do profissional (Júnior, Pleno e Sênior).

- EXPERIÊNCIA: representando a participação do profissional em projetos anteriores.

- DISPONIBILIDADE: indicando o início da disponibilidade do profissional em termos de unidades de tempo.

- DESCRIÇÃO: dados a respeito do funcionário.

É apresentado na Figura 3 a representação adotada para os funcionários, incluindo seus atributos.

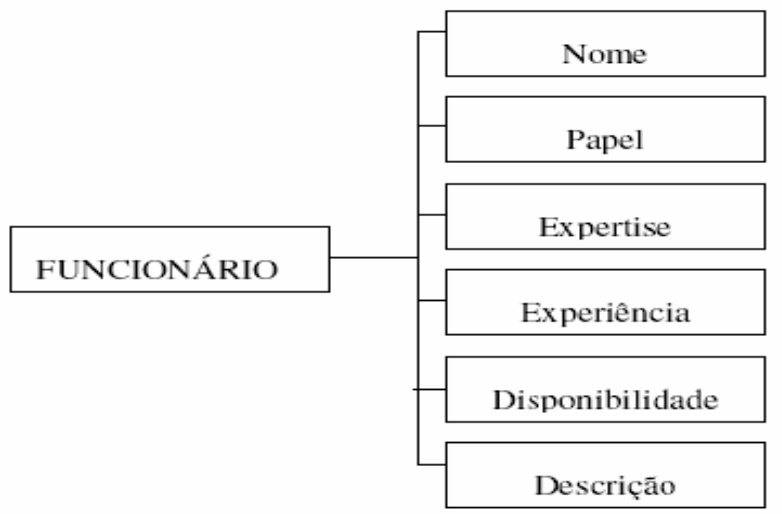

Figura 3. Representação das variáveis referentes aos funcionários.

A escolha do funcionário que executará cada atividade em determinado projeto é resultado da negociação entre os agentes que atendam ao perfil exigido. O critério para identificação do vencedor utilizado na negociação entre os agentes que se candidatarem para uma vaga, se baseia no critério de brevidade para a conclusão de cada atividade. A brevidade será calculada seguindo os seguintes critérios:

- A EXPERTISE será considerada para minimizar o tempo necessário para a execução da ATIVIDADE. Por exemplo, a EXPERTISE de Sênior reduz em 30\% o tempo de conclusão, a do Pleno reduz em 20\% e a do Júnior reduz $0 \%$.

- EXPERIÊNCIA de cada profissional em ATIVIDADES do mesmo TIPO DE PROJETO será levado em consideração para minimizar o tempo, por exemplo, 10 atividades reduziria em $5 \%$ o tempo para concluir uma atividade em um projeto semelhante e 30 atividades reduziria em $10 \%$.

- A DISPONIBILIDADE do profissional para iniciar uma nova ATIVIDADE. Os agentes poderão se candidatar a vagas mesmo sem ter encerrado um projeto atual, no entanto, isso contará negativamente durante a negociação.

Desta forma, cada agente participando de uma negociação recebe como entrada a informação de DURAÇÃO das ATIVIDADES e considera os critérios de EXPERTISE e EXPERIÊNCIA para minimizar a sua proposta e a DISPONIBILIDADE para maximizar a sua proposta. Uma vez calculada, todas as propostas de tempo para conclusão das ATIVIDADES, são comparadas, durante a negociação entre os agentes, sendo vitorioso o que apresentar a proposta de menor valor, ou seja, a mais breve. 


\subsection{Arquitetura do Sistema}

O sistema é composto por três tipos de agentes: os agentes que representam os funcionários da organização, os agentes leiloeiros que anunciam a existência de projetos e os agentes que não participam diretamente do mecanismo principal de execução. Os agentes funcionários (EmployeeAgents) possuem uma representação interna de atributos que um funcionário humano possuiria.

Os agentes leiloeiros são os iniciadores do protocolo contract-net, eles anunciam a existência de projetos e esperam a tomada de decisão dos agentes quanto à vaga no projeto. Os outros agentes são auxiliares em tarefas como a geração dos agentes e dos projetos, e a exibição dos resultados, eles são parte do sistema, mas não interferem nos protocolos de interação utilizados pelos agentes funcionários e leiloeiros.

\subsection{Exemplo do Funcionamento do Sistema}

Ao chegar um novo projeto na empresa os agentes receberão um aviso das vagas no projeto por um agente leiloeiro, negociarão entre si e concorrerão a uma vaga de trabalho no mesmo, logo após as negociações serão escolhidos os agentes que farão parte da solução final. Para ilustrar o processo de organização dos grupos é apresentado um exemplo de negociação dos agentes para a vaga de desenvolvedor.

Supondo que o projeto possui os seguintes atributos:

- TIPO DE PROJETO: Indústria.

- ATIVIDADES: Implementação de “funcionalidade”, na linguagem de programação Java.

- PERFIL: profissional com habilidades em linguagem de programação Java.

- DURAÇÃO: 10 unidades de tempo. atributos:

Supondo que os funcionários modelados como agentes possuem os seguintes

- Nome: Agente 1

- Papel: Desenvolvedor;

- Expertise: Sênior;

- Experiência: Participou de inúmeras atividades em projetos, porém, nenhuma participação em atividades nos projetos do tipo Indústria;

- Disponibilidade: Disponível para iniciar atividades a partir da terceira unidade de tempo;

- Nome: Agente 2

- Papel: Desenvolvedor;

- Expertise: Pleno;

- Experiência: Participou de trinta projetos do tipo Indústria;

- Disponibilidade: Disponível para iniciar atividades a partir da primeira unidade de tempo;

Na negociação entre os agentes, apresentada na Figura 4, o vencedor seria o Agente 2, apesar de receber uma pontuação menor pelo seu grau de expertise em comparação ao Agente 1, o Agente 2 possui uma experiência de participação em projetos do tipo indústria que o Agente 1 não possui e também está disponível para 
iniciar o projeto a partir da data de inicio, e o Agente 2 só estará disponível depois de três unidades de tempo após a data de início do projeto.

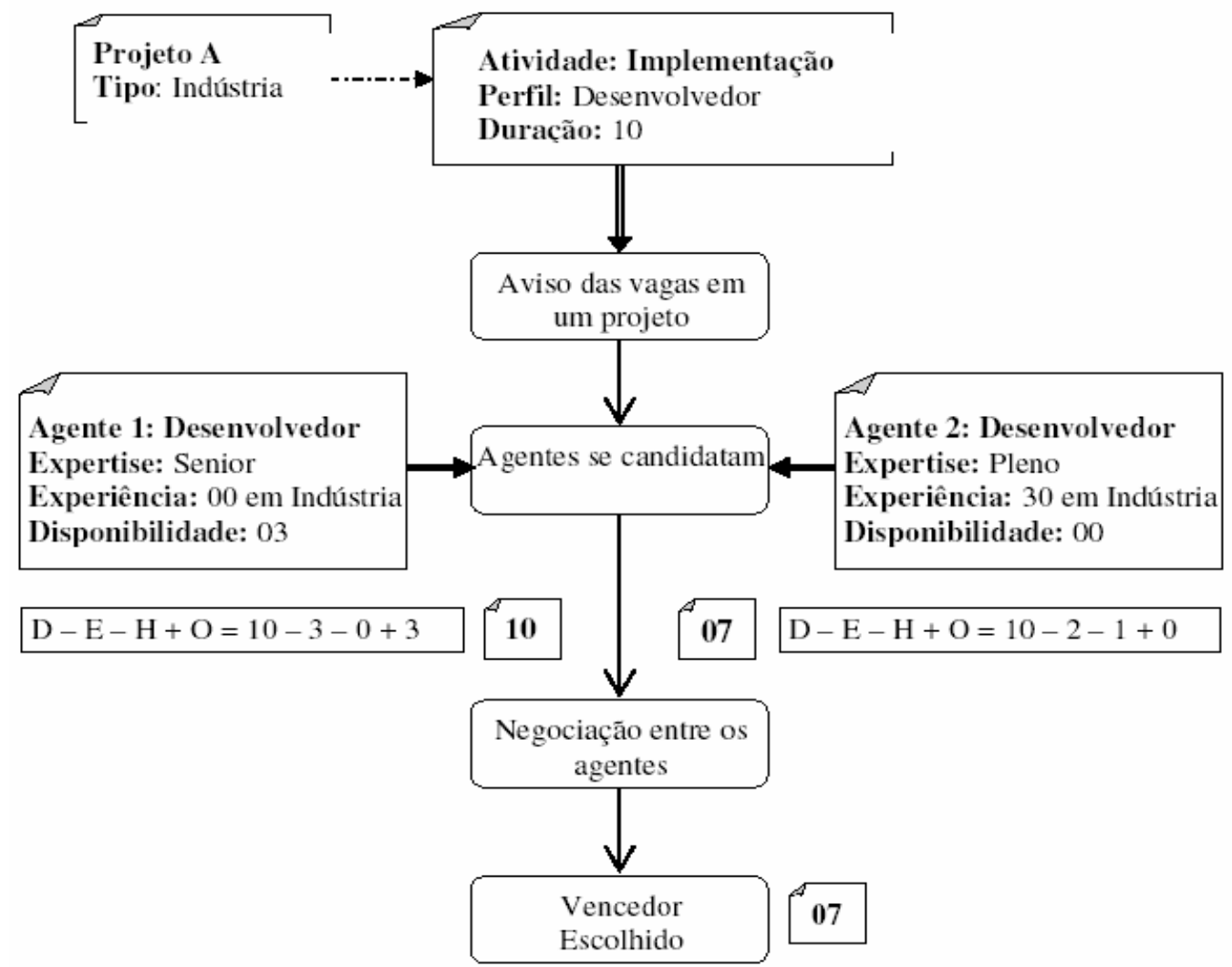

Figura 4. Diagrama de exemplo do funcionamento da negociação.

\section{Resultados}

O sistema proposto nesse artigo tem como objetivo organizar grupos de trabalho a partir de especificações de projetos. O protótipo desenvolvido utiliza um sistema MultiAgentes para representar os funcionários e o protocolo contract-net para simular a adição dos agentes nos projetos.

Os resultados obtidos são dados por heurísticas que são usadas para determinar quanto tempo uma atividade com tempo estimado seria finalizada. Elas têm por finalidade apenas comparar os agentes, por isso não devem ser considerados como valores reais. O resultado exibido pelo sistema é a composição do grupo para um determinado projeto. Cada grupo vai possuir as datas iniciais e a duração estimada. Em testes em um ambiente controlado foram obtidos os resultados apresentados na Tabela 1. Esses resultados mostram como a variação dos valores dos atributos influi no comportamento do sistema. 
Tabela 1 - Resultados obtidos nos testes realizados

\begin{tabular}{|c|c|c|c|}
\hline Variável & Valor Testado & Resultado Esperado & Resultado Obtido \\
\hline Expertise & Junior & $\begin{array}{l}\text { Não fazer parte de grupos } \\
\text { onde concorra os agentes } \\
\text { que possuam Expertise } \\
\text { Máster ou Sênior }\end{array}$ & $\begin{array}{l}\text { Não foi escolhido } \\
\text { para nenhum desses } \\
\text { grupos }\end{array}$ \\
\hline Expertise & Master & $\begin{array}{l}\text { Venceu disputas com } \\
\text { agentes com expertise } \\
\text { Junior }\end{array}$ & $\begin{array}{l}\text { Venceu disputas } \\
\text { com esses agentes }\end{array}$ \\
\hline Expertise & Sênior & $\begin{array}{l}\text { Venceu disputas com } \\
\text { agentes com expertise } \\
\text { Máster e Junior }\end{array}$ & $\begin{array}{l}\text { Venceu disputas } \\
\text { com esses agentes }\end{array}$ \\
\hline Histórico & $\begin{array}{l}\text { Variação no número de } \\
\text { unidades de tempo } \\
\text { trabalhado em atividades } \\
\text { do mesmo tipo de projeto }\end{array}$ & $\begin{array}{l}\text { Diminuição no tempo } \\
\text { estimado de acordo com o } \\
\text { aumento das unidades de } \\
\text { tempo nas atividades }\end{array}$ & $\begin{array}{l}\text { Resultado esperado } \\
\text { foi obtido }\end{array}$ \\
\hline Disponibilidade & $\begin{array}{l}\text { Aumentar a ocupação do } \\
\text { agente em mais de um } \\
\text { projeto simultaneamente }\end{array}$ & $\begin{array}{l}\text { À medida que a ocupação } \\
\text { aumenta o tempo estimado } \\
\text { também aumenta como } \\
\text { forma de penalização. }\end{array}$ & $\begin{array}{l}\text { Resultado esperado } \\
\text { foi obtido }\end{array}$ \\
\hline
\end{tabular}

\section{Conclusão e Trabalhos Futuros}

A composição de grupos de trabalho em projetos é uma atividade complexa e requer experiência e conhecimentos específicos para executar a atividade. Diversas técnicas estão disponíveis para este fim, entretanto neste trabalho propomos um sistema que realiza a alocação de indivíduos em grupos de trabalho de acordo com as atividades, perfis e duração de um determinado projeto, através de agentes.

Os resultados obtidos mostram, portanto, que o sistema proposto possibilita a formação eficiente de grupos de trabalhos em projetos cumprindo o papel ao qual se propõe constituindo-se de uma ferramenta computacional que poderá ser utilizada na tomada de decisões por gerentes de projetos.

Diversos trabalhos relacionados foram listados, entretanto nenhum deles foca no escalonamento para projetos, como abordado neste trabalho. Esta abordagem é eficiente como visto nos resultados.

Para garantir uma maior coerência dos dados seria possível coletar métricas de produtividade em projetos reais e aplicar alguma técnica de aprendizado para usar esses valores no processo de decisão dos agentes. Além disso, identifica-se como possibilidade para trabalhos futuros o tratamento de escalonamento dinâmico, onde as necessidades de um projeto vão sendo alteradas a medida que o projeto está sendo desenvolvido, tanto pelo surgimento de novas atividades ou funcionários, como pela reorganização dos prazos para execução das tarefas. 
Outra opção seria o tratamento de projetos globalmente e não mais individualmente, desta forma, o critério de escolha poderia ser ampliado levando em consideração diversos projetos ao invés de apenas um.

\section{Referências}

Bellifemine, F., G. CAIRE, POGGI, A. e RIMASSA, G. (2003), JADE - A White Paper. TILAB (Telecom Italia Lab). Turin, Italy, p.141. 2003. (3)

Chan, P. e Weil, G. (2002), Using Multiple-level Models to solve large-scale employee scheduling, http://4c.ucc.ie/ tw/ecai02/chan.ps.

Conway, R. W., Maxwell, W. L. e Miller, L. W. (1967), Teory of Scheduling, Addison Wesley, Nova York.

Daum, B. e Merten, U. (2003), System Architecture with XML, Elsevier Science, 2003,Page 441

FIPA (2002), FIPA Contract Net Interaction Protocol Specification, http://www.fipa.org/specs/fipa00029/SC00029H.pdf.

Glover, F. e McMillan, C. (1986), The general employee scheduling problem: An integration of management science and artificial intelligence. Computers and Operations Research, 13(5):563-593, 1986.

Gosling, J. e McGilton, H. (1996) “The Java Language Environment White Paper”, http://java.sun.com/docs/white/langenv/, Maio.

Gröbner, M. e Wilke, P. (2001), Optimizing Employee Schedules by a Hybrid Genetic Algorithm, Lecture Notes in Computer Science, Volume 2037, Jan 2001, Page 463

Shamblin, J. E. e Stevens, G. T. (1979). Pesquisa Operacional: Uma abordagem básica, Tradução: Carlos R. V. Araújo, Atlas, São Paulo.

Tien, J. M. e Kamiyama, A. (1982), On Manpower Scheduling Algorithms. SIAM Review, Vol. 24, No. 3 (Jul., 1982) , pp. 275-287

Welch, P. (2004) "JCSP: Communicating Sequential Processes for Java White Paper", http://www.cs.kent.ac.uk/projects/ofa/jcsp/, July.

Wooldridge, M. (2002), An introduction to Multiagents Systems, Wiley, Londres. 